\title{
Information-based Case Grammar
}

\author{
Keh-jiann CHEN \\ Institute of Information Science \\ Academia Sinica \\ Taipei, Taiwan \\ R.O.C.
}

KCHENOIS@TWNCTUOI.BITNET

\author{
Chu-Ren HUANG \\ Institute of History and Philology \\ Academia Sinica \\ Taipei, Taiwan \\ R.O.C.
}

HSCHUREN@TWNAS\$86.BITNET

\begin{abstract}
In this paper we propose a framework of Informationbased Case Grammar (ICG). This grammatical formalism entails that the lexical entry for each word contain both semantic and syntactic feature structures. In the feature structure of a phrasal head, we encode syntactic and semantic constraints on grammatical phrasal patterns in terms of thematic structures, and cncode the precedence relations in terms of adjunct structures. Such feature structures denote partial information which defines the set of legal phrases. They also provide sufficient information to identify thematic roles. With this formalism, parsing and thematic analysis can be achieved simultaneously. Due to the simplicity and flexibility of Information-based Case Grammar, context dependent and discontinuous relations such as agreements, coordinations, long-distance dependencies, and control and binding, can be easily expressed. ICG is a kind of unfication-based formalism. 'Therefore it inherits the advantages of unification-based formalisus and more.
\end{abstract}

In the feature structure of a phrasal head, we cncode syntactic and semantic constraints on grammatical phrasal patterns in terms of thematic structures, and encode the precedence relations in terms of adjunct structures. The feature structure of a potential phrasal head denotes partial information for defining the set of legal/grammatical phrises. It also provides enough information to identify the thematic roles for arguments and adjuncts [Chen 89]. In other words, with ICG, parsing and thematic analysis are achieved simultaneously without additional operation; and generation with thematic structure can be done with the identical formalism.

We take Mandarin Chinese as our representational target. Thus, the features were selected to account for Chinese only. However, the abstract design of this formalism is not limited to only the representation of Chinese. Since the Chinese lexicon is impoverished in inflection, it is necessary to fully stipulate both semantic and syntactic information for the purpose of both parsing and generation. Furthermore the precedence relationship of constituents is defined over thematic roles. This seems to be more appropriate for Chinese. By coincidence, Bresnen and Kanerva's [Bresnan 89] lexical mapping theory represents a shift towards the possibility of semantics major approaches.

\section{Feature Structures for Mandarin Chinese}

Chinese is a weakly marked language with no inflection. Nevertheless, the linear order of arguments and adjuncts are relatively free. Hence syntactic-only representations would cause tremendous ambiguities. For representational precision and for parsing adecuacy, semantic information is indispensible. The most important semantic information includes 1 . argument structures and their semantic restrictions, and 2. the semantic fealures for each word which are necessary to identify thematic roles. Hence the following feature structure (1) was sclected so that each lexical entry can be uniformly represented by the same structure with lexically or syntactically defined value (including null).

(1)

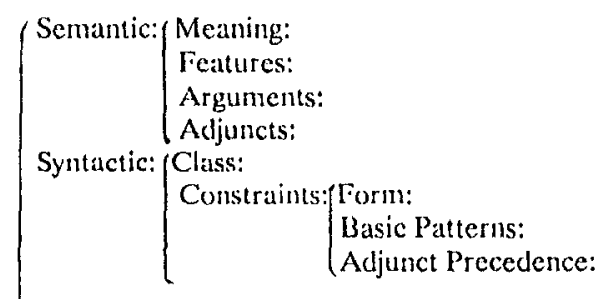

A typical example (2) is given here before we introduce and summarize the values for each feature path below. 
(2) Chiwan "persuade":

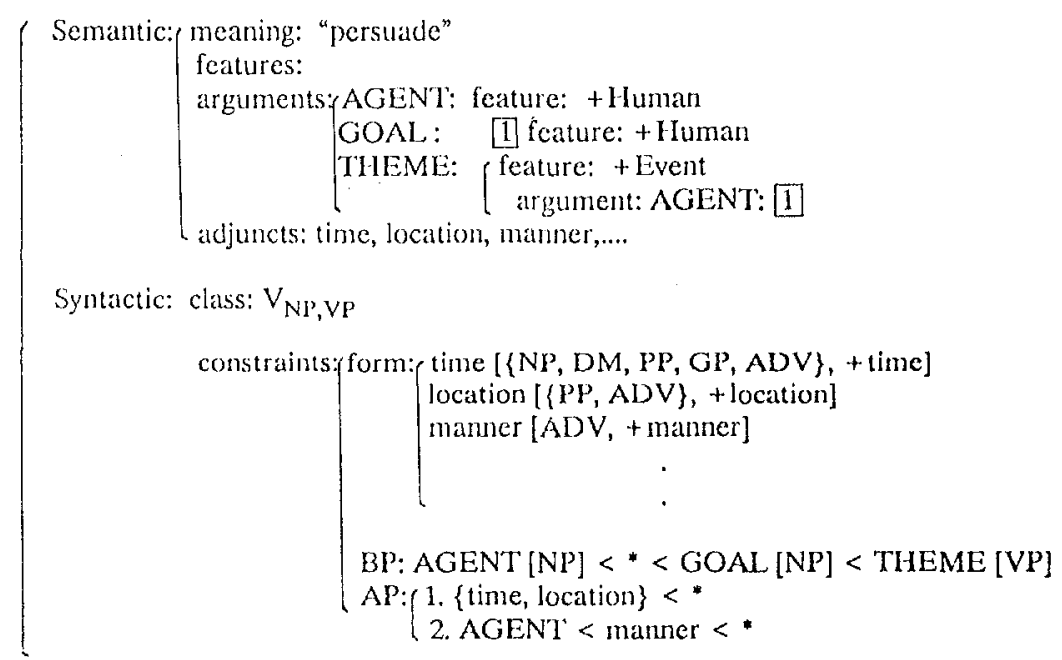

Meaning: an atomic value denoting the meaning of the word.

Features: a set of atomic values which are the semantic features of the word; e.g. + animate, + physical.

Arguments: at set of thematic arguments for the head if the word is a possible phrasal head; null otherwise. The value for each thematic argument is a feature structure of the same type as the value for the feature patt 'scmantic'

E.g, 'a nice boy' is an agent, as in 'A trice boy drove the cattle.'

\section{(3) $\triangle \mathrm{GLNT}$ :}

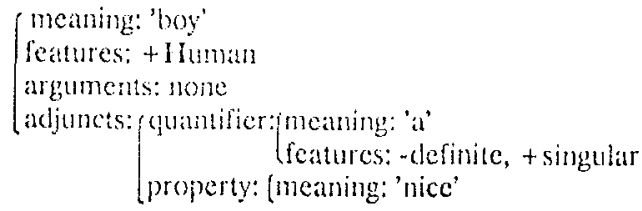

Argument structures for a verb are equivalent to case frames and case restrictions of this verb [Fillmore 68, Winograd 83]. Case restrictions indicate semantic preferences of thematic roles and function as a gudide to identifying each case role [Chen 89]. The argument structures for other pharasal heads such as prepositions, post-positions and conjunctions serve similar purposes.

Adjuncts: a set of permissible adjuncts of the head word. The value of each adjunct is a feature structure of the same type as arguments.

E.g. 'yesterlin'

(4) time: meaning: 'yesterday'

features: + time

arguments: none

adjuncts: none
Arguments, adjuncts and head form the right hand side of an immediate dominance rule.

Syntactic Class: atomic values denoting the syntactic class of the word.

The syntactic class of a word serves two purposes. The first is to denote the syntactic type. The second is as an index for inheriting common syntactic properties belonging to the mother node in the syntactic hierarclly.

Syntactic Form: a set of syntactic and semantic definitions for arguments and adjuncts.

The synlactic form for each thematic role in fact constrains the syntactic structures and semantic features of this role. We consider semantic restriction as part of the syntactic constraint. For instance, temporal expressions are instantiated by at least live different syntactic categories which are nouns phrase (NPS), compounds with determinatives and measures (DM, such as $\mathrm{Sam}$ dicul Shi-fen threc-hours ten-minute, i.e. 'three(en'), post-position phrases (GPs), preposition phrases (PPs), or adverbs (ADVs). They all share a common semantic feature + lime regardless of their catcgories. Therefore the temporal expressions can be expressed as time [\{NP, DM, $\mathrm{PP}, \mathrm{GP}, \mathrm{ADV}\}$, + time]. For complex expressions, we adopt the choice system used by Halliday in the systernic grammar [Winograd 83].

13asic Patterns: a set of linear precedence rules governing arguments and heads.

The basic patterns for verls denote the possibie sentential patterns, including optional argument onission. The familiar syntactic generalizations with regard to passivization, topicalization etc. can be captured by lexical rules [Gazdar 85 , Pollard 87 , Sells 85]. Adopting the lexical mapping theory, however, is a thcoretical possibility to make basic patterns obsolete [Bresnan 89, Huang 89]. 
Adjunct Precedence: a set of linear precedence and cooccurrence constraints for adjuncts.

The following notations were adopted.

$$
\begin{aligned}
& * \text { denotes a phrasal head. }
\end{aligned}
$$

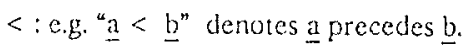

$$
\begin{aligned}
& <\text { : e.g. "a } \ll \text { b" denotes } \underline{\text { a }} \text { imnediately } \\
& \text { precedes b. }
\end{aligned}
$$

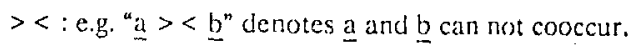

$$
\begin{aligned}
& \{\}: \text { e.g. }{ }^{\text {a }}<\{\underline{b}, \underline{c}\}^{n} \text { denotes a precedes both } \mathrm{b} \text { and }
\end{aligned}
$$

(5) A nice boy persuaded John to go to school yesterday.

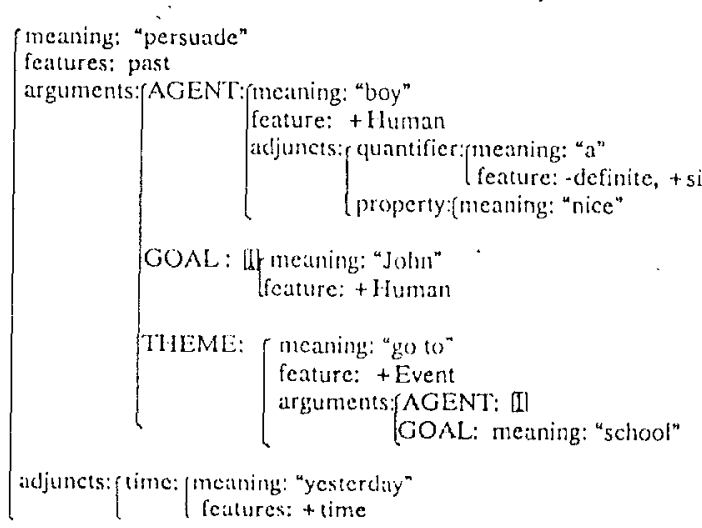

\section{The Formal Definition for Information-based Case Grammar: The Lexicon and Principles}

The ICG is composed of two major components. One is the lexicon which is the set of feature structures as described in section 2. The other is the principles. Each feature structure can be viewed as a set of interpretable representations of syntactic and scmantic information governed by a formal syntax. A parser or generator interpretes feature struclures while parsing or generating sentences. The interpretation processes are guided by the principles of the grammar. The principles define wellformedness conditions and the rules for information management for sentences and phrases. The parser or generator takes lexical information and unify it in such a way that changes of lexical information would not affect the phrasing or generating process. Therefore we claim that ICG is declarative. The major principles of ICG are summarized below:

\section{1) Head Driven Principle}

The feature structure of a head contains the partial information that defines the permissible set of phrases with it as a phrasal head. The possible head types and

\begin{tabular}{|c|c|}
\hline Hcad & Pirase \\
\hline $\bar{V}$ & $S, V P$ \\
\hline$N$ & NP \\
\hline Prep & $P p$ \\
\hline Post P & Gil \\
\hline Determinimt & $\begin{array}{l}\text { DM (delerminative } \\
\text { and metsurecompound) }\end{array}$ \\
\hline Conjunction & $\mathrm{CP}$ (conjunctive phrase) \\
\hline
\end{tabular}
respective phrase types for Mandarin Chinese are as follows.

(6)

The syntactic classes of the heads determine the syntactic types of their projections. VP is defined as an $S$ without subject [Gazdar 87]. 
2) Well-formedness Conditions

Like LFG [Bresnan 82], we have the following well-formedness conditions:

a. Completeness and functional biuniqueness conditions,

b. Coherence conditions,

and $\mathrm{c}$. Linear precedence and syntactic form constraints.

In fact, the completeness condition is enforced with respect to Basic Patterns (BP) only. The cases of argument omission are idiosyncratically determined by verb classes, and are not governed by the completeness condition. On the other hand, adjuncts are optional and constrained only by linear precedence rules AP and form restrictions. The functional uniqueness condition is also relaxed a bit to account for cases of multiple occurrences of some adjuncts such as modal at the sentential level, and property on the noun phrase level, by annotating Kleene's star on top of the adjunct modal and the adjunct property.

3) Feature Propagation Principles

a. Head feature convention [Gazdar 85]

b. Foot feature principle [Gazdar 85],

c. Explicit feature paths:

Explicitly denoting the daughter's feature in the mother node by a feature path such as the 'argument feature',

d. Conditional features:

Locally ambiguous semantic features can be expressed by conditional features. For instance, the preposition bei 'by' can mark an agent, a causer, or an instrument depending upon whether the arguments are animate, nonplysical, or physical inanimate respectively. Therefore the feature structure of bei 'by' would be (7).

(7) Bci 'by'

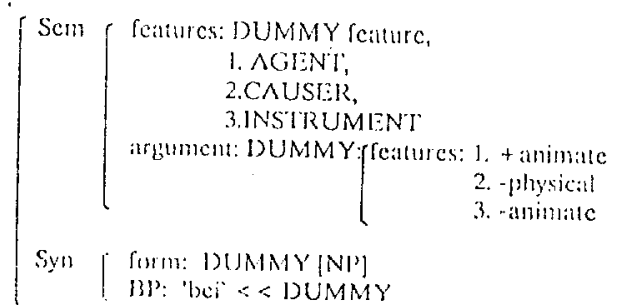

There are many possibie types of semantic features allowed in ICG. They are differentiated by attribute value pairs. The three required types of semantic features for Chinesc are 1 . scmantic classes e.g. + animate 2, syntactic/semantic feature, e.g. + NEG 3. thematic roles, e.g. AGENT. Different types of features can be identificel simply by their attribute names c.g. we know AGENT in <ARGUMENT AGENT> is a thematic rolc.

The head feature principle is the same as the one in GPSG [Gazdar 85] which states that in any local subtrec, the head features of the mother are identical to the head features of the head daughter. Similarly, our Foot feature principle also follows GPSG. Roughly speaking, foot features are passed up from any daughter in a tree, with the upper and lower limits of this propagation are determined by prior specification [Gazdar 85]. In Chinese, + Question, + Negation, + Plural, + Definite etc. are all considered foot features.

The semantic class of a thematic role is usually determined by its head daughter. However, for the marked cases with the syntactic categories of $\mathrm{PP} / \mathrm{GP}$, the semantic classes are determined by complement daughters. We can not define every semantic type as a foot feature. Therefore, we propose to explicitly state the daughter's feature in the mother node by a feature path such as 'DUMMY features' in (7).

\section{What Makes ICG a Good Representational Language}

We think that the simplicity and flexibility of the ICG formalism makes it a good representational language. It is simple since ICG is a type of context-free grammar and the altributes for feature structures are uniform for all different types of phrases.

Preparation of lexical feature structures are straightforward. Linguists can start with the categorial feature structure of each entry by discovering idividual idiosyncracies and then modifying the categorial feature structure accordingly. As for flexibility, ICG is much more flexible than the other contexi-free grammatical forms such as BNF, GPSG, etc., due to the wider scope of accessibility and the richness of information encoding on each thematic constituent. Context dependent and discontinuous relations such as agreenents, coordinations, long-distance dependencies, control and binding, can be easily expressed in ICG.

\subsection{Agreement and Coordination}

Agreement and coordination pose similar problems in represcentation (but different problems in processing). Both have to express the relations between daughters. Coordination can be viewed as the agreement of syntactic or/and semantic classes between two daughter arguments of a conjunction. In contrast, other types of agrecments are the relations between head daughter and complement daughters. Since ICG provides an explicit accessing capability to the daughters features, both types of agreements can be casily solved. The only difference is that coordination requires a variable ranging over a finite domain of symactic classes and/or semantic classes to denote the common features of two arguments (8). For instance, the subjcet verb agreement problems are solved by stating agreement constraints on each subject role in every basic pattern. For example, the AGENT of the verb "persuades" is expressed as AGENT $[N P,+$ singular, + third] in active voice.

(8)

$$
\left\{\begin{array}{l}
\text { meaning : "and" } \\
\text { feature : DUMMY feature } \\
\text { arguments: DUMMY t: feature: class } x
\end{array}\right.
$$

DUMMY 2: feature: class $x$ 


\subsection{Long-distance Dependency}

Kaplan and Zaenen [Kaplan 88, Kaplan 89] proposed functional uncertainty as a new descriptive technique, assuming grammatical function in LFG, in order to account for longdistance dependencies. The schema of functional uncertainty avoid the problem of infinite specifications so that the attribute positions for topicalizable constituents could be realized as a regular cxpression such as $\left(\uparrow\right.$ comp ${ }^{*}$ subj|obj $)=(\uparrow$ topic $)$. This equation expresses the uncertainty about what the with-in clause functional roles of an extraposed topic might be. It offered a clearer and more accurate characterization of long-distance dependencies but still could not handle the case of context dependency in topicalization. For instance, there are many verbs in Chinese which do not allow the object to be topicalized. When such a verb is the head of an embedded sentence, it is clear that (comp obj) is not toplicalizable either. There is no way to predict the topicalizable constituent in depth by way of regular expressions. There are similar examples in English.

(9)

a. Who $i$ did Mary think that Bill saw $e_{i}$ ? ? Who $i$ did Mary quip that Bill saw $\mathrm{e}_{i}$ ?

b. Who ${ }_{i}$ did John tell you (that) Mary thought that Bill saw $e_{i}$ ?

?Who, did John tell you (that) Mary quipped that Bill saw $\mathrm{e}_{i}$ ?

However we can solve such context dependent problems in ICG by recursively defining the topicalizable constituents in terms of topicalizable constituents of embedded sentences. The verb with sentential complements usually causes the problems. We may encode the topicalized sentence patterns as a part of BP or as derived by lexical rules. The topic of the embedded senience is one of the topicalizable constituents of such verbs. For instance the topicalized sentence pattern of example (2) is:

\section{TOPIC [TOPIC (THEME)]< AGENTINP]< *} GOAL[NP] < THEME/TOPIC

The topics of embedded sentences are defined recursively under the sentence palterns of the verbs of embedded sentences. Detailed discussion is given in [Chen 90, Huang 90].

\subsection{Control and Binding}

Functional control is the relation that exists between an antecedent and the missing subject in an XCOMP or XADJUNCT [Bresnan 82, Sells 85]. The coindex label adopted in the unification-based grammars is a simple solution to such problems. We use the same scheme in ICG, e.g. in (2). Anaphoric binding was solved in LFG by the concept of $\mathrm{F}$. command [Bresnan 82, Sells 85]. The same concept is also applicable to ICG.

\section{Conclusion}

From the brief sketch above, it is clear that each lexical entry contains a large amount of redundant syntactic and semantic information shared by other entries belonging to the same category. Hence, a more elegant and economic strategy is to form a catcgory hierarchy and to store shared information on higher level nodes. Each lexical entry contains only individual idiosyncracies. Thus the redundancy in representation can be removed and data consistency can also be easily maintained.

ICG is a kind of unification-based formalism. Therefore all the advantages of unification-based formalism were kept in ICG. Furthermore, additional advantages are incorporated via the following design fcatures of ICG.

\section{Declarative}

The lexical information defines legal sentences or phrase. And the changes in the above information does not affect processing procedures and results.

\section{Algorithm-free}

Different control processes are allowed for parsing or generation. Regardless of whether it is sequential, parallel, or heuristic control, the result will be the same.

3. Suitable for parallel processing

The processes are head driven. Each possible head can initiate a phrasal construction process. Thus, processes can be executed in parallel.

\section{Allows a partial parse}

At any moment of the unification processes, the accumulated information shows the partial result up to that moment even if the input is ungrammatical.

5. Semantic structure is universal

The semantic information contained in the feature structures of each lexical entry is universal. Different languages differ with regard to syntactic of information.

\section{Incorporated with thematic role identification scheme}

The parametrical information for identifying thematic roles can be encoded in ICG such that syntactic parsing and semantic analysis can be done in parallel.

\section{Parsing result is a thematic structure}

Recent theoretical linguistic studies are concerned with the relationship between thematic structures and argument structures (e.g. Brensan and Kanerva 1989). Our formalism dircetly and explicitly represents thematic structures.

Last but not least, we expect future studies of ICG to confirm the plausibility of the following advantages.

\section{Efficient parsing}

ICG has the advantages of lexicon-based parsing which is better focused on the relavant syntactic and semantic information.

2. Cognitive reality

The language capability of a man seems to be improved daly after day by learning and polishing lexical information. ICG is able to reflect this phenomenon. 


\section{Generation power}

ICG provides at way of gencrating surface sentences from thenatic structures. The pragmatic comsideration of the selection of the sentence patterns can be accomplished by incorporating the pragmatic features in the basic patterns and lexical rules.

\section{Acknowledgement}

Research of this paper was partially supported by NSC grant 778-0408-10001-001 and the Electronic Research and Service Ofganization, Industrial Technology Research Institute, Iaiwan, R.O.C. under Contract No. X2-79007. Some of the cxamples are taken from the research results of the Chinese Knowledge Processing Group at the Computing Center of Academin Sinica. We alone, of counse, are responsible for any possible errors contained in this paper.

\section{Rejerences}

Bresnan, J., 1982. The Mental Representation of Grammatical Relations, Cambricige: Mir I'ress.

Bresnan, J. and J. Kanerva, 1989. Locative Inversion in Chichewa: A Case Study of Factorizaton in Grammar.

Lingustic Inoury 20, pp. $1-50$.

Chen, K. J. and C. S. Cha, 1988. The Design of a Conceptual structure and lis Relation to the Parsing of Chinese Sentences. ICCPCOL'88, Toronto.

Chot, K.J, C.R. Hunng, and I.P. Chang, 1989. The Identification of thematic Roles in Parsing Mandarin Chinese, Procedings of ROCLING II (1989), Tapei, Taivan, $\mathrm{pp}, 121-146$.

Chen, K.J. C.R. Hang, and VV.P. Chen, 1990. Resolution of Long-Distance Dependencies with Recursive Information Enbeding, In preparation.

Iiilmore, C, 1968. The Case for Case. In L. Bach and R. Harms (I:ls.), Universals in Iinguistic Theory, New York: llolt, Rinebart, and W'inston.

Gazdar, G. et al. 1987. Category Structures. CSLI report 102, Stanford: Coner for the Study of Language and Information.

Gazdar, G., I: Klein, G.K. Pullum, and I.A. Săg, 1985. Generabized Phase Structure Grammar. Cambridge: Blackwell, and Cambridge, Mass.: Harvard University Press.

Iuang, C.R. 1989. Mandarin Chinese and the lexical Mapping Theory. The 1989 International Conference on Sino-Tibetan Languages and Linguistics. Hawaii.

Huang, C.R., K.J. Chen, W.P. Chen and T.Y. Hu, 1900. Resolution of Long-Distance Dependences in Mandanin Chinese-With an Algorithm Based on Functional Uncertainty. To appear in the Proceedings of the 1990 Anternational Conference on Computer Processing of Chinese and Oriental Languages (ICCPCOL'90).
Kaplan, R.M. and J. Maxwell, 1988. An Algorithm for Functional Uncertainty. Proceedings of Coling '88, Budapest, 297-302.

Kaplan, R.M. and A. Zaenen, 1989. Long Distance Dependencies, Constituent Structure, and Functional Uncertainty. In M. Baltin \& A. Kroch (Eds.), Alternative Conceptions of Phrase Structure. Chicago: Chicago University Press, 17-42.

Karttunen, L., 1986. Radical Lexicalism, CSLJ Report No. CSL1-86-68, Stanford: Center for the Study of Language and Information.

Mellish, C.S., 1988. Implementing Systemic Classification by Unification, Computational Linguistics, Vol. 14 \#1, 40-51.

Pollard, C. and I. Sag, 1987. Information-based Syntax and Semantics, Vol. I. Fundamentals, CSLI Lecture notes \# 13, Stanford: Center for the Study of Language and Information

Sells, P., 1985. I ectures on Contemporary Syntactic Ihcories. CSLI Lecture Notes no. 3. Stanford: Center for the Study of Language and Information.

Shieber, S.M., 1986. Introduction to Unification-based Approaches to Grammar. Stanford: Center for the Study of Language and Information.

Tomita, M., 1986. Efficient Parsing for Natural Langlage, Boston: Kluwer Academic.

Uszkoreit, Il, 1986. Categorial Unification Grammars. In Proceedings of Coling 1986. Bomn: University of Bom. Also appeared as Report No. CSLI-86-66, Stanford: Center for the Study of Language and Information.

Winograd, 'T., 1983. Language as a Cognitive Processes, Vol. 1, Syntax, Adtison-Wesley. 